\title{
Sequence-defined multifunctional polyethers via liquid-phase synthesis with molecular sieving
}

Ruijiao Dong ${ }^{1}$, Ruiyi Liu ${ }^{1}$, Piers R. J. Gaffney ${ }^{1}$, Marc Schaepertoens ${ }^{1}$, Patrizia Marchetti ${ }^{1}$, Christopher M. Williams $^{2}$, Rongjun Chen ${ }^{1}$ and Andrew G. Livingston ${ }^{1} *$

${ }^{1}$ Department of Chemical Engineering, Imperial College London, South Kensington Campus, London, SW7 2AZ, UK. ${ }^{2}$ EPSRC UK National Mass Spectrometry Facility (NMSF), Swansea University Medical School, Singleton Park, Swansea, SA2 8PP UK. *e-mail: a.livingston@imperial.ac.uk

Synthetic chemists have devoted tremendous effort towards the production of precision synthetic polymers with defined sequences and specific functions. However, the creation of a general technology that enables precise control over monomer sequence, with efficient isolation of the target polymers, is highly challenging. Here, we report a robust strategy for the production of sequence-defined synthetic polymers through a combination of liquid phase synthesis and selective molecular sieving. The polymer is assembled in solution with real time monitoring to ensure couplings go to completion, on a three-armed star-shaped macromolecule to maximise efficiency during the molecular sieving process. This approach is applied to the construction of sequence-defined polyethers, with sidearms at precisely defined locations that can undergo site-selective modification after polymerisation. Using this versatile strategy, we have introduced structural and functional diversity into sequence-defined polyethers, unlocking their potential for real-life applications in nanotechnology, healthcare and information storage.

Natural macromolecules, such as nucleic acids and proteins, are heteropolymers with perfectly defined chain length, monomer sequence and chirality. This precise control of the primary sequence provides structural and functional diversity sufficient to generate the molecular complexity required by all living organisms ${ }^{1,2}$. Polymer chemists have employed strategies such as single monomer insertion ${ }^{3,4}$, tandem monomer addition ${ }^{5}$, kinetic control ${ }^{6}$, segregated templating ${ }^{7,8}$, and sequential growth polymerisation ${ }^{9,10}$, to provide polymers with narrowly disperse, but not uniform, chain lengths and approximately controlled sequences. Nevertheless, these sequence-controlled approaches cannot compete with the precision of nature. To prepare truly uniform sequence-defined polymers, iterative synthesis can afford the required nature-like degree of control over the final sequence. In iterative synthesis specific monomers are added one-at-a-time to the end of a growing polymer chain, reaction debris is then separated from the chain extended polymer, and the cycle is repeated using the next monomer in the sequence ${ }^{11,12}$. Solid-phase iterative synthesis ${ }^{13}$ is the premiere method for preparation of sequence-defined polymers, mainly because of the simple reaction and purification processes (i.e. filtration and washing), as well as its ease of automation ${ }^{14}$. However, the insoluble solid supports are often expensive, and the purity of the growing polymer is not readily monitored during synthesis ${ }^{7,12}$. Furthermore, the rates of solid-phase coupling reactions are limited by diffusion into the solid support, ultimately leading to a decline in coupling yields and accumulation of deletion errors ${ }^{15}$. Moreover, solid-phase synthesis is generally difficult to scale up, precluding many industrial applications, particularly in materials science ${ }^{7,10,12}$.

Consequently, liquid-phase iterative synthetic methods have long been proposed to overcome these limitations, benefiting from unrestricted liquid-phase kinetics, straightforward sampling for analysis, and well understood principles for scale-up ${ }^{12}$. In recent years, a number of liquid-phase methodologies have been exploited which allow access to a wider range of backbone chemistries, and more precise control over monomer sequence and side-chain functionality. For example, Whiting ${ }^{16,17}{\text {. } \text { utz }^{18-20}, \text { Hawker }^{21,22}, \text { Meier }^{23,24}, \text { Alabi }^{25,26} \text {, Sawamoto }}^{27,28}$ and many others ${ }^{29-}$ 
${ }^{33}$ have presented pioneering works on the synthesis of defined sequence polymers in solution. These reports are uniformly built around established chemistries with rapid coupling rates (including polyamides, polyesters, thiol-ene click, azide-alkyne click, homologation of boronic esters), unlike Williamson ether synthesis, so that high chain extension yields and sequence purities are assured, but the nature of the final polymers is secondary. Furthermore, many require tedious chromatographic purification after each synthesis cycle.
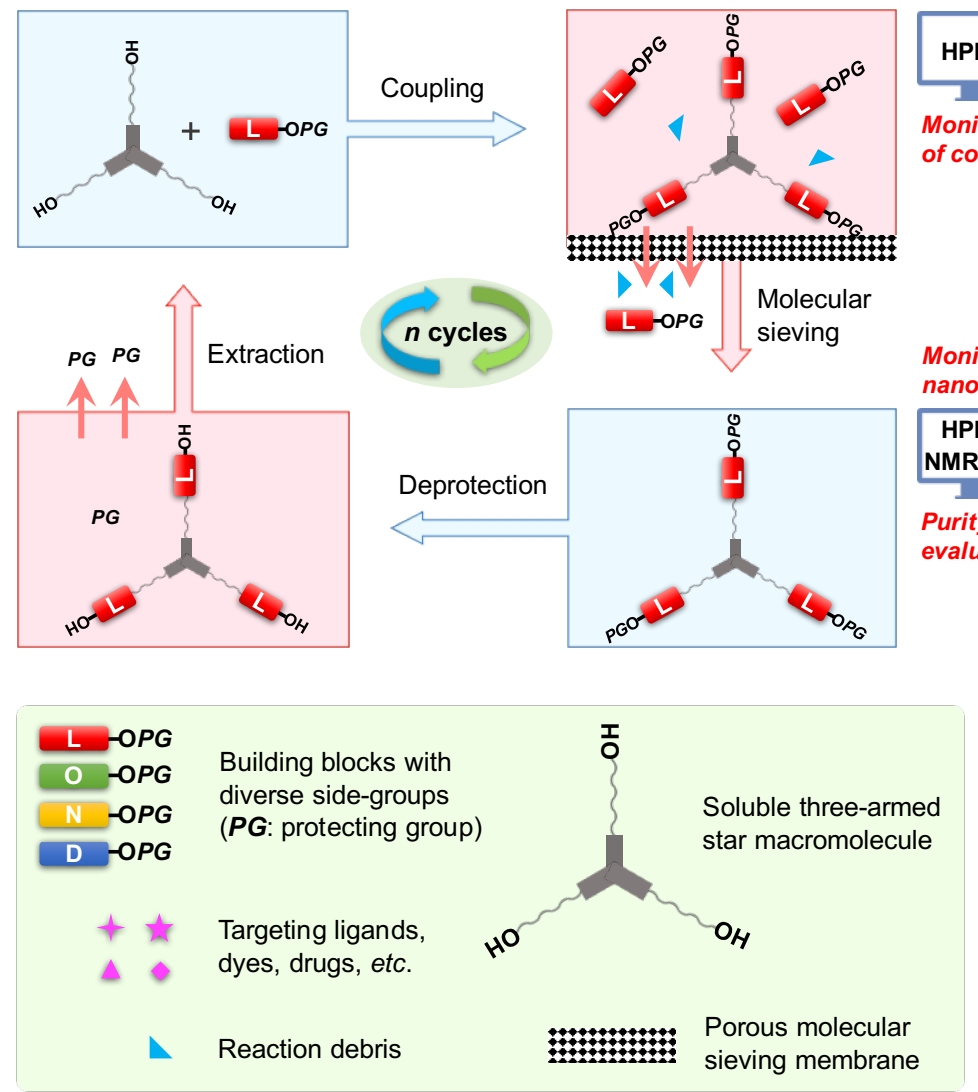

b

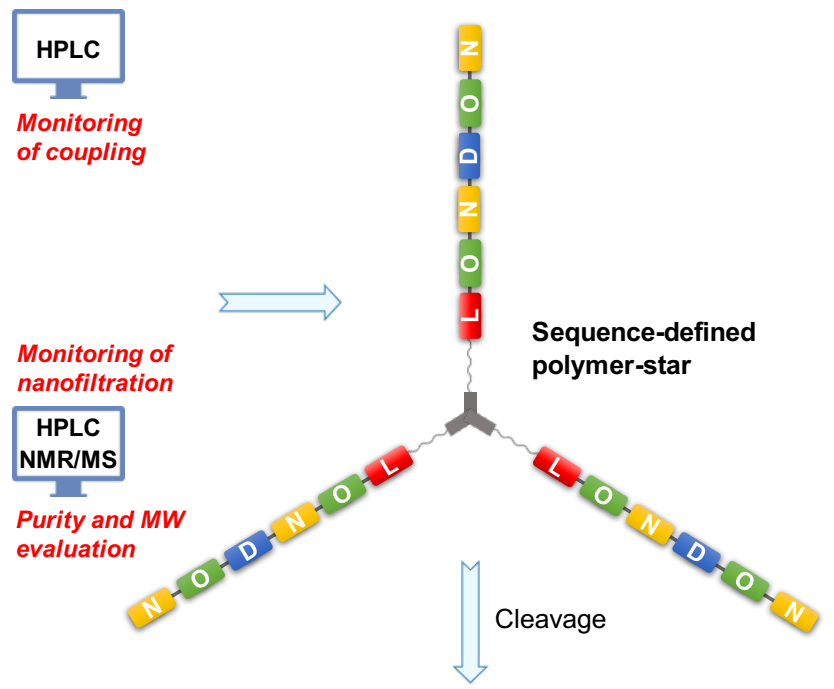

C

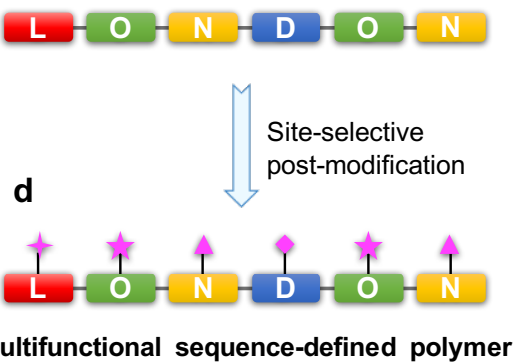

Fig. 1 | Liquid-phase synthesis with molecular sieving to prepare multifunctional sequence-defined polymers. a, Chain extension cycle: coupling of protected building blocks to the polymer-star, built around a three-armed hub; membrane separation to remove excess building block and other reaction debris; deprotection of the chain termini and extraction. During the cycle, chain extension and molecular sieving processes are monitored in real time by HPLC, then the growing species are fully characterised by NMR and MS to validate the synthesis. $\mathbf{b}$, Sequencedefined polymer-star after $n$ synthesis cycles. c, Cleavage of the polymer-star from the hub, and unblocking of any side-arm protecting groups. d, Site-selective side-arm modification to generate a multifunctional sequence-defined polymer.

Polyethers have attracted enormous interest from synthetic chemists, materials scientists and biologists owing to their excellent water solubility, biocompatibility and stealth properties ${ }^{34,35}$. Of these, discrete/uniform poly(ethylene glycols) (PEGs) with exactly defined chain lengths and excellent biocompatibility have received the most attention from synthetic chemists ${ }^{36-39}$. However, it has proved difficult to identify insoluble supports suitable for effective solidphase synthesis. A recent study has illustrated the limitations of solid phase synthesis of linear ethylene glycol (Eg) oligomers $\left(\mathrm{Eg}_{n}, n \geq 16\right)$ via the Williamson ether synthesis ${ }^{40}$. Instead, uniform linear polyethylene glycol (PEG) homopolymers have been constructed using liquid-phase iterative strategies ${ }^{36-39}$, which have usually depended on chromatographic separation to isolate the growing polyethers after each iteration of the synthesis cycle. To the best of our knowledge, the physical and biological advantages of PEGs have never been combined with the structural and 
information-bearing complexity of sequence-defined functional heteropolymers. With the exception of facile $\alpha, \omega$ chain terminal modification, polyethers have not been amenable to site-selective structural modification, optimization of inherent properties, or later incorporation of functional elements at specific sites. Therefore, the creation of a general and versatile technology for the production of multifunctional, sequence-defined synthetic polyethers with tunable side-arm functionalities directed to real-world applications is highly challenging and timely.

Herein, we describe the synthesis of a new class of multifunctional, sequence-defined polyethers that shows potential as a biomedical vehicle. These have been fabricated using a unique combination of liquid-phase iterative synthesis and selective molecular sieving (Fig. 1). Four chiral $\alpha, \omega$-end-functionalised pentagol monomers with sidearms were prepared (5-8, Fig. 2), to which we assign coding letters $\mathbf{N}, \mathbf{O}, \mathbf{L}$ and $\mathbf{D}$, respectively. Synthetic polyethers were constructed by repeated etherification of the monomers growing out from a central three-armed macromolecule (hub) (9, Fig. 2), to give a sequence-defined polyether-star (10-21). This process allows chain extension to proceed with liquid-phase kinetics, and enables facile monitoring of coupling reactions in real time by HPLC to ensure that coupling is complete. After cleavage of the arms from the hub, and deprotection of the side-arms, site-selective postpolymerization modification of the polyethers was undertaken (Fig. 6). The use of liquid-phase reactions coupled to real time monitoring and size-exclusion molecular separation technology ${ }^{41-44}$ make this platform a particularly efficient approach to the production of high purity sequence-defined polyethers (Supplementary Table 14-1). Furthermore, the introduction of reactive functional groups on the side-arms along the polyether backbone enables precise control over structure and physicochemical properties, and allows for fast and precise site-selective multifunctionalisation at a later stage $e^{45}$. This strategy offers opportunities to introduce structural versatility and further diversity into sequencedefined synthetic polyethers, thereby unlocking their potential for widespread applications ranging from healthcare and nanotechnology to information storage.

\section{Results and discussion}

Iterative polymer synthesis consists of a cycle of two reactions, repeated to the desired sequence length: chain extension by adding a single protected building block (BB) to one end of the growing polymer, followed by removal of a temporary protecting group ( $\boldsymbol{P} \boldsymbol{G}, \mathbf{F i g} .1)$, where $\boldsymbol{P} \boldsymbol{G}$ prevents uncontrolled chain extension. For the synthesis of a multifunctional polyether, the first requirement is that all molecular features on the growing polyether-star are compatible with Williamson etherification conditions. The second is that all other features are orthogonal to removal of $\boldsymbol{P G}$. At the center of the star we selected a benzylic ether linkage between the polyether chains and the hub molecule that, whilst inert to etherification conditions, may be cleaved by hydrogenolysis or Lewis acids. We then selected a hydrophilic tetrahydropyran-1-yl (Thp $=\boldsymbol{P G})$ acetal for temporary protection of the chain termini because it is very stable during etherification, but is rapidly deprotected by mildly acidic solvolysis and readily removed by simple extraction. A range of Thp-protected building blocks (BBs, 5-8) was then designed for compatibility with these primary elements, using reductive processes to deprotect reactive functionalities on the side-arms.

Because each side-arm introduces a new stereo-center into the PEG backbone, the synthesis of BBs has to be enantio-selective (Fig. 2). 4,4'-Dimethoxytriphenyl chloride (Dmtr-Cl) was reacted with either commercial $(R)$-1benzyl glycerol, or (R)-1,2-dihydroxydecane, to give chiral 1-Dmtr-ethers 1 and 2, respectively; notably, the opposite enantiomer of each BB can also be prepared using this route. The 2-OH of 1 was then etherified with desymmetrised tetragol (3), followed by additional side-arm functional group and protecting group manipulation, to afford pentagol toluene sulfonate building blocks with reactive side-arms including 5 (BnO-BB, N), $6\left(\mathrm{~N}_{3}-\mathrm{BB}, \mathbf{O}\right)$ and 7 (PmbS-BB, L) on multi-gram scales in good overall yield (Fig. 2 and Supplementary Scheme 6-1). Pentagol 8 (Octyl-BB, D) with a hydrophobic side-arm, was prepared in a similar fashion starting from 2 and 4 (Fig. 2 and Supplementary

Scheme 7-1). 

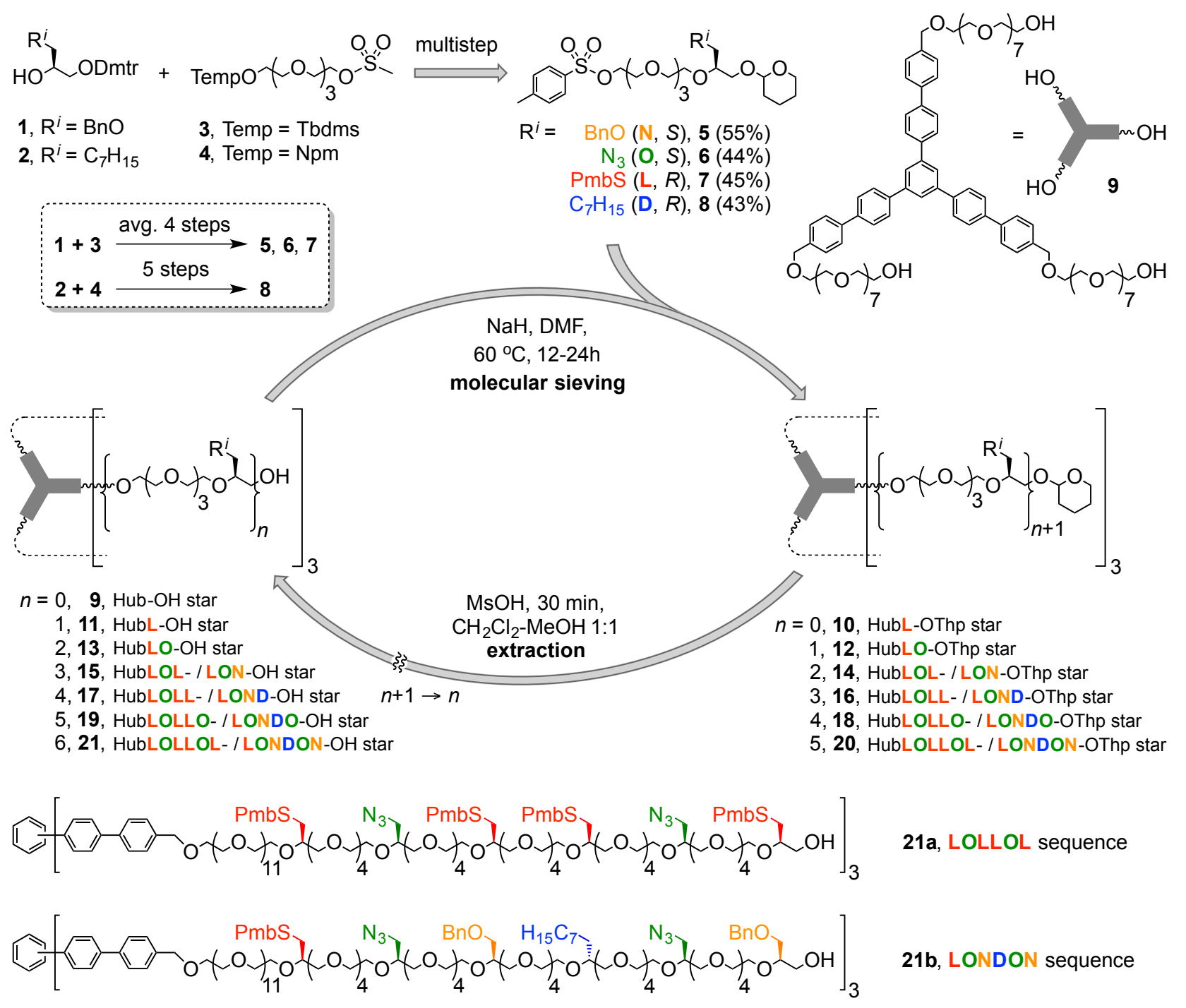

Fig. 2 | Synthesis of sequence-defined polyether-stars. Pentagol monomers (5-8) were constructed from tetragol and $(R)$-1-benzyl glycerol, or (R)-1,2-dihydroxydecane. Defined sequence polyethers with functional side-arms were assembled by repeated cycles of etherification of monomers 5-8 with polymers attached to a soluble three-armed hub 9, followed by Thp-deprotection. The intermediate polyether-stars (10-21) were purified in high yield without chromatography, alternating molecular sieving after coupling, and extraction after Thp-deprotection. Dmtr, 4,4'-dimethoxytriphenyl; Tbdms, tert-butyldimethylsilyl; Npm, naphthyl-2-methyl; Bn, benzyl; Pmb, p-methoxybenzyl.

In order to perform chromatography-free, liquid-phase polyether synthesis, a technology is required to separate reaction debris from the growing polymer. We selected molecular sieving by organic solvent nanofiltration (OSN) ${ }^{41-}$ ${ }^{43}$ for this role because of its fundamental simplicity, and the high efficiency now achievable. However, that efficiency depends upon the separation factor $\left(\beta_{A / B}\right.$, see SI for definition) for separating the desired product from reaction debris, which in turn depends upon the relative difference in molecular size of the solutes. To this end, the growing polyether was supported on a bulky three-armed hub molecule, bearing flexible octagol spacers $\left[\mathrm{Hub}\left(\mathrm{OEg}_{8}-\mathrm{OH}\right)_{3}, 9\right]^{46}$ (Supplementary Scheme 8-1). Molecular modelling (Supplementary Figs 13-1 and 13-2) suggested that a threearmed star provides a good combination of molecular bulk and steric accessibility, compared to two-armed and fourarmed alternatives. The hub of the three-armed star usefully functions as both a protecting group for one end of the polyether, and as a label for monitoring reactions because of its high UV extinction coefficient. However, the primary 
role of the three-armed hub is effectively to trimerise the growing polyether, and therefore to triple its size. Combined with the large diameter of the rigid central unit, this greatly increases the size of the target polymer. Hence, permeation of the large polyether-star through the membrane is minimized compared to the relatively small BBs, and separation efficiency is maximized (Fig. 3a).
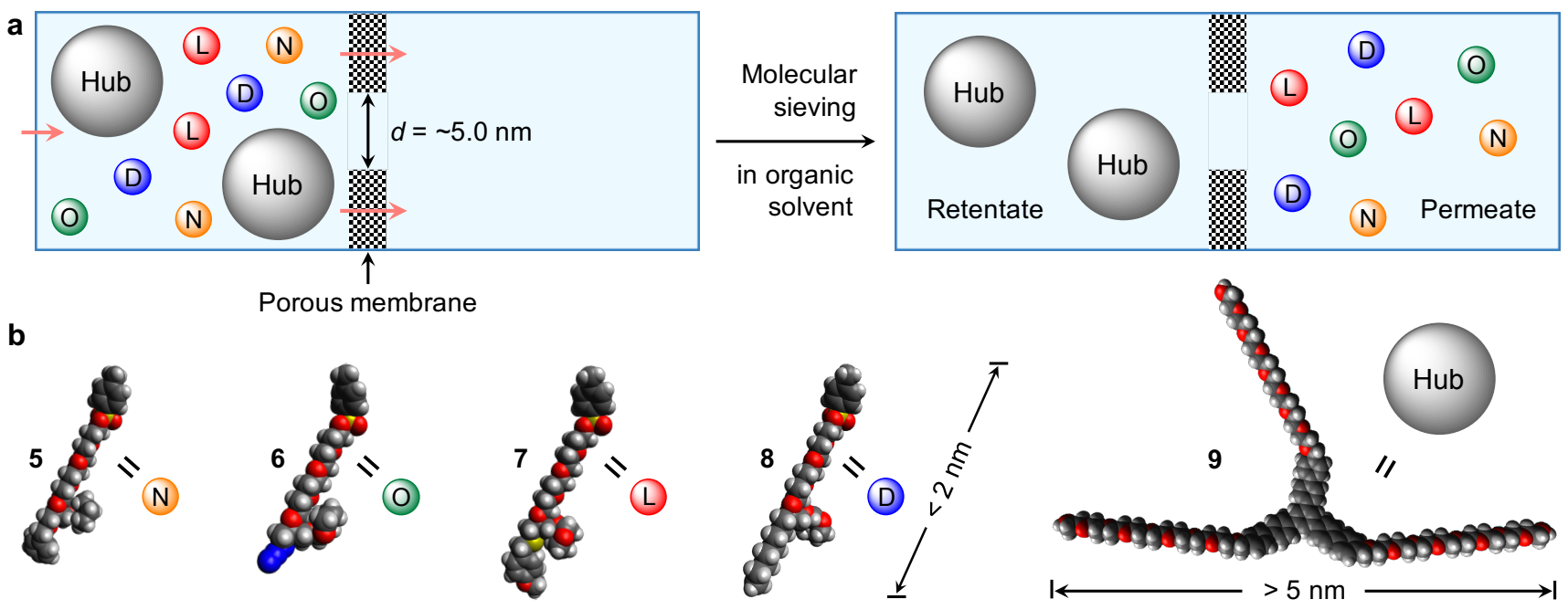

C

d

e
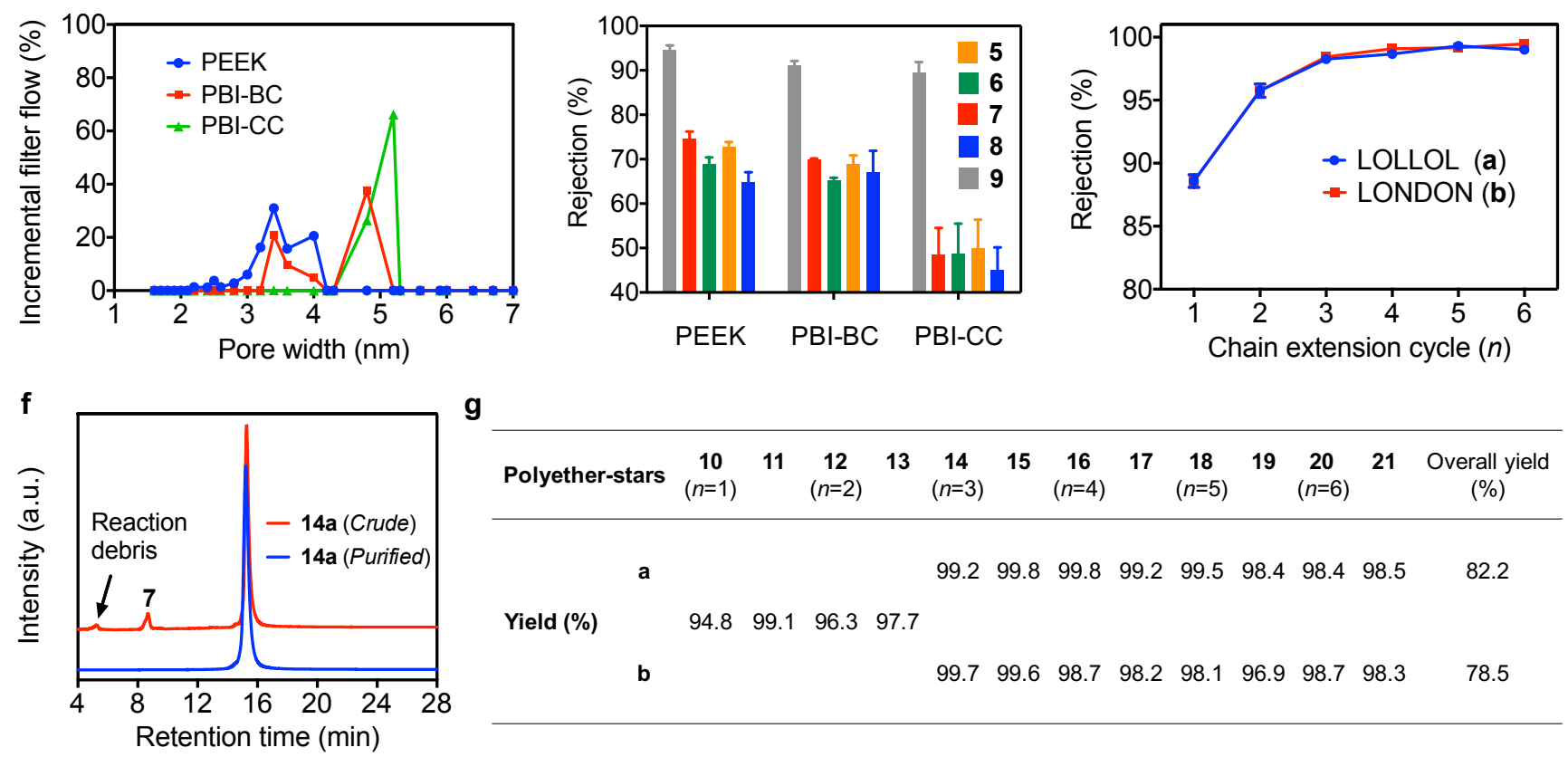

g

\begin{tabular}{|c|c|c|c|c|c|c|c|c|c|c|c|c|c|}
\hline Poly & $\begin{array}{r}10 \\
(n=1\end{array}$ & 11 & $\begin{array}{c}12 \\
(n=2)\end{array}$ & 13 & $\begin{array}{c}14 \\
(n=3\end{array}$ & 15 & $\begin{array}{c}16 \\
(n=4\end{array}$ & 17 & $\begin{array}{c}18 \\
(n=5)\end{array}$ & 19 & $\begin{array}{c}20 \\
(n=6)\end{array}$ & 21 & $\begin{array}{c}\text { Overall yield } \\
(\%)\end{array}$ \\
\hline
\end{tabular}

a

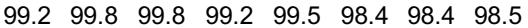

82.2

Yield (\%) $94.8 \quad 99.1 \quad 96.3 \quad 97.7$

$\begin{array}{llllllllll}\text { b } & 99.7 & 99.6 & 98.7 & 98.2 & 98.1 & 96.9 & 98.7 & 98.3 & 78.5\end{array}$

Fig. 3 | Size-exclusion molecular sieving and determination of separation efficiency. a, Schematic representation of molecular sieving of pentagol BBs (5-8) and three-armed tris(octagol) hub (9). b, Space-filling models of BBs (5-8) and hub (9). Colour code of the space-filling model: C, grey; N, blue; $\mathrm{H}$, white; O, red; S, yellow. c, Pore size distributions of PEEK, PBI-BC and PBI-CC asymmetric membranes determined by liquid-liquid porometry. d, Rejection of BBs (5-8) and hub (9) for PEEK, PBI-BC and PBI-CC in THF-MeOH (4:1), data represent mean value $(n=4)$. e, Rejection of all polyether-star intermediates in the LOLLOL- and LONDON-star syntheses, data represent mean value $(n=$ 4). f, Typical HPLC profiles of a polyether-star (14a) before and after membrane purification. $\mathbf{g}$, Yield of all polyether-stars after each synthesis cycle. PEEK, polyether ether ketone membrane; PBI-BC, a bench-cast poly(benzimidazole) (PBI) membrane, cross-linked with $\alpha, \alpha$ '- $p$ dibromoxylene (DBX) and modified with Jeffamine-M-2005; PBI-CC, a continuously-cast PBI membrane, cross-linked with DBX and modified with Jeffamine-M-2005. 
We required a membrane for molecular sieving of the crude reaction mixtures. This membrane should withstand organic solvents, acids, bases, and various reactive species. Three robust asymmetric membranes were prepared: one from polyether ether ketone (PEEK) ${ }^{47}$; another from a bench-cast poly(benzimidazole) (PBI), cross-linked with $\alpha, \alpha^{\prime}-$ p-dibromoxylene (DBX) and modified with Jeffamine-M-2005 (PBI-BC); and a final one from continuously cast PBI, cross-linked with DBX and modified with Jeffamine-M-2005 (PBI-CC) ${ }^{48}$. The membranes' pore size distributions were determined by liquid-liquid porometry (Fig. 3c). The average pore size of PBI-CC was around $5.0 \mathrm{~nm}$, significantly larger than those of PEEK (ca. $3.5 \mathrm{~nm})$ or PBI-BC $(c a .4 .0 \mathrm{~nm})$. The molecular weights of the BBs (5-8) ranged from 531 to $642 \mathrm{Da}$, and their sizes were all less than $2 \mathrm{~nm}$, whilst the hub (9), with a molecular weight of 1,682 Da, had a diameter of more than $6 \mathrm{~nm}$ (Fig. 3b). In principle, all of these membranes might be expected to retain the bulkier hub, and even more so the growing polymer-stars, whilst allowing the smaller BBs to permeate (Fig. 3a). Of the three membranes, PBI-CC showed the highest solvent permeance $\left(12 \mathrm{~L} \cdot \mathrm{m}^{-2} \cdot \mathrm{h}^{-1} \cdot \mathrm{bar}^{-1}\right)($ Supplementary Fig. 42), and importantly the highest separation factor $\left(\beta_{H u b / B B}=5.0\right)$ between the hub (9) and BBs (5-8) in THF-MeOH (4:1), compared to either PEEK $\left(\beta_{H u b / B B}=4.5\right)$ or PBI-BC $\left(\beta_{H u b / B B}=3.4\right)$ (Fig. 3d). Changing the solvent to MeCN$\mathrm{MeOH}(1: 1)$ further enhanced the separation factor with PBI-CC up to 7.7 (Supplementary Fig. 4-4), although the solvent permeance was reduced to $3 \mathrm{~L} \cdot \mathrm{m}^{-2} \cdot \mathrm{h}^{-1} \cdot \mathrm{bar}^{-1}$ (Supplementary Fig. 4-3). These results are more than sufficient to ensure rapid and efficient separation of the growing polymer-star from reaction debris by molecular sieving through the PBI-CC membrane in $\mathrm{MeCN}-\mathrm{MeOH}(1: 1)$.

To commence polyether-star synthesis (Fig. 2), the first BB (6 equiv. PmbS-BB, 7, single letter code L) was coupled to the tris-octagol hub (9), under typical etherification conditions ( $\left.\mathrm{NaH}, \mathrm{DMF}, 60^{\circ} \mathrm{C}, 12-24 \mathrm{~h}\right)$. For practical OSN separation of the subsequent reaction mixtures, it is critical that the desired polymer-star is isolated in high yield at each iteration cycle. However, the rejection values for small polyether-stars are significantly below 100\% (Fig. 3e), so that diafiltration using a single-stage membrane system would give low stepwise yields. To this end, a two-stage membrane diafiltration system has been developed and was used throughout this work, greatly increasing the product recovery from each round of nanofiltration ${ }^{49}$. Excess PmbS-BB (7) was readily removed by membrane nanofiltration, passing through the PBI-CC membrane with other reaction debris, while the bulkier polyether-star $\mathrm{Hub}\left(\mathrm{OEg}_{8} \mathbf{L}\right.$ $\mathrm{OThp})_{3}(\mathbf{1 0})$ was highly retained by the two-stage membrane system (see SI). The growing polyether-star was then washed from the membrane apparatus and deprotected (catalytic $\mathrm{MsOH}$ in $\mathrm{CH}_{2} \mathrm{Cl}_{2}-\mathrm{MeOH}$ 1:1, 30 min). The resultant $\mathrm{Hub}\left(\mathrm{OEg}_{8} \mathbf{L}-\mathrm{OH}\right)_{3}(\mathbf{1 1})$ was purified by simple extraction ready for the next round of chain extension.

After 6 iterations, two sequence-defined polyether-stars were prepared. In the first, just two BBs were combined in a palindromic sequence to prepare $\mathrm{Hub}\left(\mathrm{OEg}_{8} \mathbf{L O L L O L - O H}\right)_{3}(\mathbf{2 1 a})$ in $82 \%$ overall yield ( $>98 \%$ purity). In the second, all four BBs, PmbS-BB $(7, \mathbf{L}), \mathrm{N}_{3}-\mathrm{BB}(\mathbf{6}, \mathbf{O}), \mathrm{BnO}-\mathrm{BB}(\mathbf{5}, \mathbf{N})$ and Octyl-BB $(\mathbf{8}, \mathbf{D})$, were added in sequence to give the intermediate $\mathrm{Hub}\left(\mathrm{OEg}_{8} \mathbf{L O N D}-\mathrm{OH}\right)_{3}(\mathbf{1 7} \mathbf{b})$ in $88 \%$ cumulative yield after 4 cycles, and finally $\mathrm{Hub}\left(\mathrm{OEg}_{8} \mathbf{L O N D O N}-\mathrm{OH}\right)_{3}$ (21b) in 79\% yield (>96\% purity) over 12 steps (i.e. 6 synthesis cycles) (Fig. 3g). As the polymer-stars grew in length, over the initial three cycles it was found that their rejection increased from $c a .90 \%$ to ca. 99\%, and held constant thereafter (Fig. 3e). The enhanced rejection with increasing size of the growing Thpprotected polyether-stars led to greater isolated yields with each successive cycle, eventually approaching $100 \%$. These are much higher than conventional chromatographic purification (Supplementary Table 15-1), whilst the yield of deprotection/extraction remained constant at $97-99 \%$ (Fig. 3g).

During each synthetic cycle (i.e. coupling, molecular sieving and deprotection), all steps were monitored by HPLC. The progress of the critical etherification reaction could be monitored in real time because, in all cases, the ThpO-polyether-stars had 1-2 min longer retention time than the starting HO-polyether-stars (Figs 4a-d). In cases where the reaction was found to be incomplete, further reactants were added to drive it to completion, assuring a high 
purity of final polyether-stars. The coupling reactions for the first three chain extension cycles were complete within $12 \mathrm{~h}$, but for the last three cycles the reactions took longer (18-24 h), so more monomer was added (up to 12 equiv., i.e. 4 equiv. per arm). Monitoring molecular sieving of the crude reaction mixtures by HPLC revealed that the quantity of residual monomers in the apparatus decreased steadily with the duration of diafiltration, finally becoming undetectable (Fig. 3f and Supplementary Figs 5-3 to 5-12). Thp deprotection was also monitored by HPLC, where the retention time again reduced by 1-2 min. All the intermediates up to the LOLLOL-star gave single HPLC peaks both with Thp on (Fig. 4a) and Thp off (Fig. 4b), demonstrating the high purity of the polyether-stars. The retention time was observed to lengthen with the growth of the polyether-stars. The LONDON-star intermediates exhibited similar patterns in their HPLC profiles (Figs 4c-d). GPC was further used to characterise all growing polyether-stars, giving single GPC peaks and so verifying their uniform nature (Supplementary Fig. 16-1). Concomitant with their growth, the GPC retention time of the polyether-stars gradually shortened, demonstrating a stepwise increase in molecular size / molecular weight. With the incorporation of the hydrophobic Octyl-BB (8, D) onwards shoulders were observed on the HPLC peaks for all the following intermediates. MALDI-ToF-MS verified that these polyetherstars with octyl side-arm (16b-21b) are very pure, with no obvious impurity detected (Fig. 4i and see SI). Therefore, the shoulders are attributed to an HPLC artefact due to poor solubility of the octyl-containing polyether-stars in HPLC eluent (an $\mathrm{MeOH}$-water mixture).
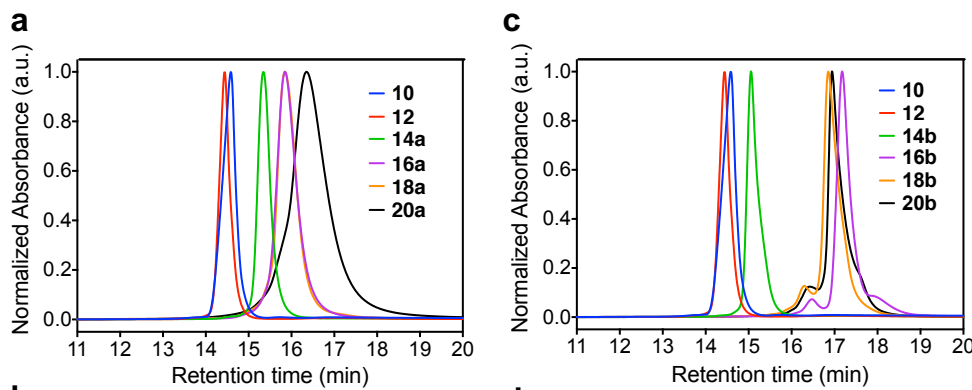

\begin{tabular}{|c|c|c|c|}
\hline \multirow{2}{*}{$\begin{array}{l}\text { Polyether } \\
\text {-stars }\end{array}$} & \multirow{2}{*}{ Sequence } & \multicolumn{2}{|c|}{$m / z[M+N a]^{+}$} \\
\hline & & Calculated & Observed \\
\hline 10 & $\operatorname{Hub}\left(\mathrm{OEg}_{8} \mathrm{~L}-\mathrm{OThp}\right)_{3}$ & 3115.6 & 3115.6 \\
\hline 11 & $\mathrm{Hub}\left(\mathrm{OEg}_{8} \mathrm{~L}-\mathrm{OH}\right)_{3}$ & 2863.4 & 2863.4 \\
\hline 12 & $\operatorname{Hub}\left(\mathrm{OEg}_{8} \text { LO-OThp }\right)_{3}$ & 3942.0 & 3942.0 \\
\hline 13 & $\mathrm{Hub}\left(\mathrm{OEg}_{8} \mathrm{LO}-\mathrm{OH}\right)_{3}$ & 3689.9 & 3690.0 \\
\hline $14 a$ & $\operatorname{Hub}\left(\mathrm{OEg}_{8}\right.$ LOL-OThp $_{3}$ & 5101.6 & 5101.5 \\
\hline $14 b$ & $\mathrm{Hub}\left(\mathrm{OEg}_{8} \mathrm{LON} \mathrm{OThp}\right)_{3}$ & 4963.6 & 4963.6 \\
\hline $15 a$ & $\mathrm{Hub}\left(\mathrm{OEg}_{8} \mathrm{LOL}-\mathrm{OH}\right)_{3}$ & 4849.4 & 4849.3 \\
\hline $15 b$ & $\mathrm{Hub}\left(\mathrm{OEg}_{8} \mathrm{LON}-\mathrm{OH}\right)_{3}$ & 4711.4 & 4711.4 \\
\hline $16 a$ & $\mathrm{Hub}\left(\mathrm{OEg}_{8} \mathrm{LOLL} \mathrm{OThp}\right)_{3}$ & 6261.1 & 6261.1 \\
\hline $16 \mathrm{~b}$ & $\mathrm{Hub}\left(\mathrm{OEg}_{8}\right.$ LOND-OThp $_{3}$ & 5960.4 & 5960.4 \\
\hline $17 a$ & $\mathrm{Hub}\left(\mathrm{OEg}_{8} \text { LOLL-OH }\right)_{3}$ & 6007.9 & 6008.0 \\
\hline $17 \mathrm{~b}$ & $\mathrm{Hub}\left(\mathrm{OEg}_{8} \text { LOND-OH }\right)_{3}$ & 5708.2 & 5708.2 \\
\hline $18 a$ & $\operatorname{Hub}\left(\mathrm{OEg}_{8}\right.$ LOLLO-OThp $_{3}$ & 7084.5 & 7084.2 \\
\hline $18 \mathrm{~b}$ & $\mathrm{Hub}\left(\mathrm{OEg}_{8}\right.$ LONDO-OThp $_{3}$ & 6786.8 & 6787.2 \\
\hline $19 a$ & $\mathrm{Hub}\left(\mathrm{OEg}_{8} \mathrm{LOLLO} \mathrm{OH}\right)_{3}$ & 6834.4 & 6834.6 \\
\hline $19 b$ & $\mathrm{Hub}\left(\mathrm{OEg}_{8} \mathrm{LONDO}-\mathrm{OH}\right)_{3}$ & 6533.6 & 6534.0 \\
\hline $20 a$ & 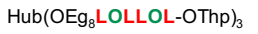 & 8245.1 & 8246.0 \\
\hline $20 \mathrm{~b}$ & 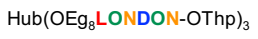 & 7807.4 & 7808.0 \\
\hline 21a & $\operatorname{Hub}\left(\mathrm{OEg} \mathrm{E}_{8} \mathrm{LOLLOL-OH}_{3}\right.$ & 7992.9 & 7993.0 \\
\hline 21b & 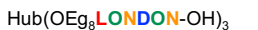 & 7551.2 & 7551.0 \\
\hline
\end{tabular}
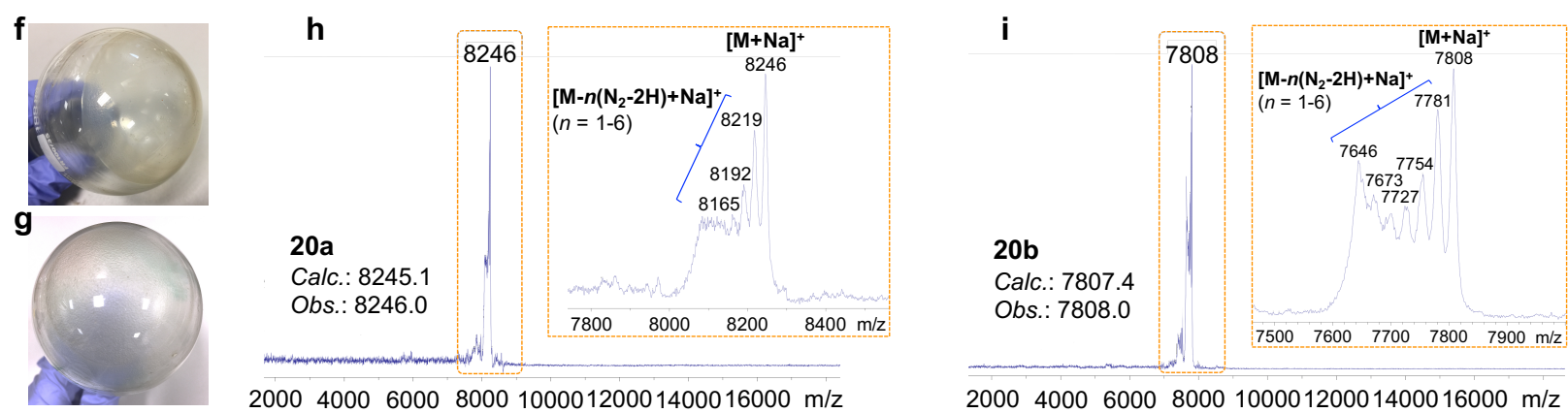

$200040006000800010000120001400016000 \mathrm{~m} / \mathrm{z}$

Fig. 4 | Characterisation of all polyether-star intermediates. a-b, HPLC traces of all LOLLOL polyether-stars (a, Thp-protected; b, deprotected). c-d, HPLC traces of all LONDON polyether-stars (c, Thp-protected; d, deprotected). e, MALDI-ToF-MS characterisation of all sequence-defined polyether-stars (10-21). f, Photograph of the pristine sample of 20a. g, Photograph of the pristine sample of 20b. h, MALDIToF-MS of 20a. i, MALDI-ToF-MS of 20b. 
The molecular structures of all growing polyether-stars were verified by ${ }^{1} \mathrm{H}-,{ }^{13} \mathrm{C}-$, and $2 \mathrm{D}-\mathrm{NMR}$, plus MALDIToF-MS (Fig. 4e and see SI). For the final LOLLOL-star (20a), both types of side-arms could be separately detected, with the expected relative integrations for the characteristic resonances from the hub and Thp acetal. Two distinct classes of chiral centers were distinguishable along the PEG backbone by ${ }^{1} \mathrm{H}_{-}{ }^{13} \mathrm{C}$-HSQC correlation spectroscopy (Supplementary Fig. 9-1). Similarly, for the final LONDON-star (20b), all four different stereo-centers along the PEG backbone could be picked out by ${ }^{1} \mathrm{H}-{ }^{13} \mathrm{C}-\mathrm{HSQC}$ correlation spectroscopy (Supplementary Fig. 9-2). MALDIToF-MS further confirmed the identity of the final 20a and 20b. In Fig. 4h, the observed $m / z$ value (8246.0) of the expected molecular ion $[\mathrm{M}+\mathrm{Na}]^{+}$for $\mathbf{2 0 a}$ was consistent with the calculated molar mass of $8245.1 \mathrm{Da}$. Notably, fragmentation peaks were observed just below the molecular ion at $m / z$ 8084-8219 due to loss of molecular nitrogen from six azide groups $\left[\mathrm{M}-n\left(\mathrm{~N}_{2}-2 \mathrm{H}\right)+\mathrm{Na}\right]^{+}(n=1-6)$. Apart from the expected MALDI-ToF-MS molecular ion for 20b at $m / z$ 7808, a very similar fragmentation pattern to 20a was also seen at $m / z$ 7646-7781 indicative of six azide moieties (Fig. 4i). Notably, despite the shoulder in the later HPLC chromatograms (Fig. 4c), there was no sign of polyetherstar contaminants with deletion sequences.

The final ThpO-polyether-stars (20a and 20b) were isolated as pale yellow, viscous oils on $>200 \mathrm{mg}$ scale (Fig. 4f-g), and were fully soluble in most organic solvents (e.g. $\mathrm{MeOH}, \mathrm{MeCN}, \mathrm{Me}_{2} \mathrm{CO}$, THF, $\mathrm{CH}_{2} \mathrm{Cl}_{2}, \mathrm{CHCl}_{3}$, DMF and DMSO). Interestingly, the resulting HO-polyether-stars (21a and 21b) were readily dispersed in water to form stable milky colloidal solutions due to their amphiphilic nature (Supplementary Fig. 18-2). Furthermore, the DSC and TGA data for 21a and 21b (Supplementary Figs 17-1 and 17-2) revealed the marked effect of side-chain structure and monomer sequence on their thermal properties. In particular, the glass transition temperatures $\left(\mathrm{T}_{\mathrm{g}}\right)$ for oily sequencedefined PEG 38-mer-stars (21a and 21b) were $-58.4{ }^{\circ} \mathrm{C}$ and $-52.7^{\circ} \mathrm{C}$ respectively (Supplementary Fig. 17-1), which were much lower than that of semi-crystalline linear PEGs, suggesting that the side-chain structure greatly impacted the intermolecular packing of the PEG backbone.

a

21

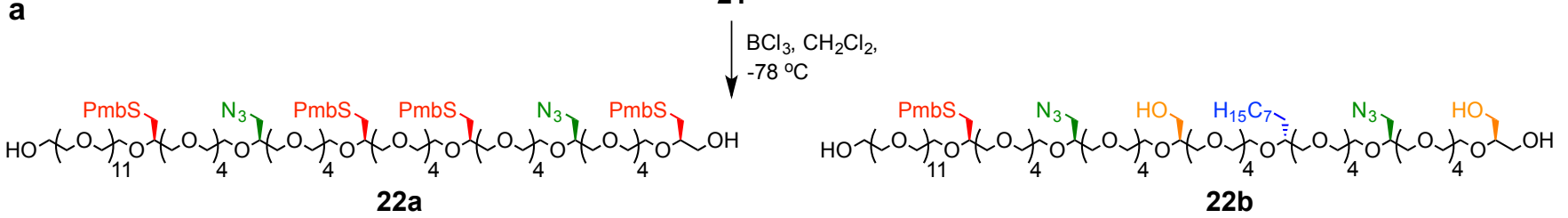

b
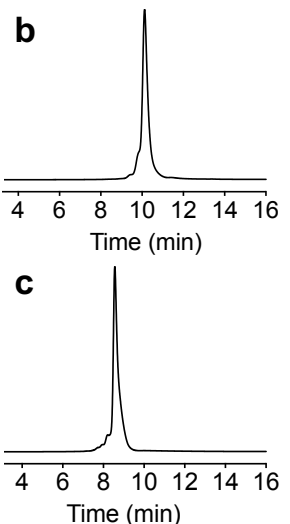

d

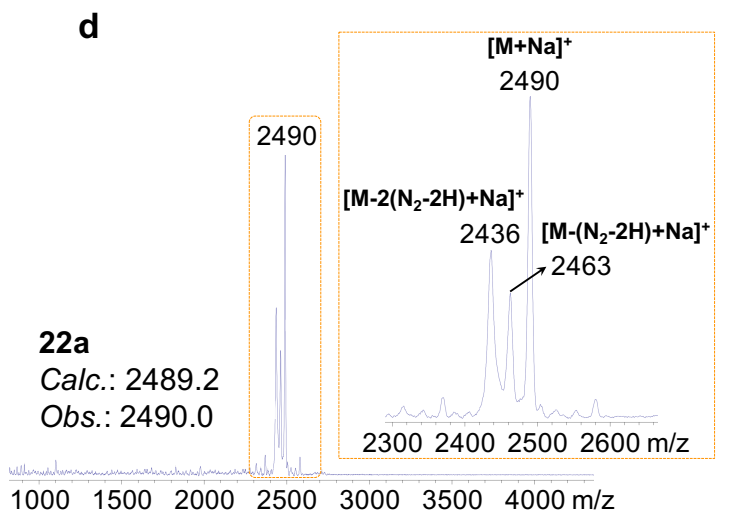

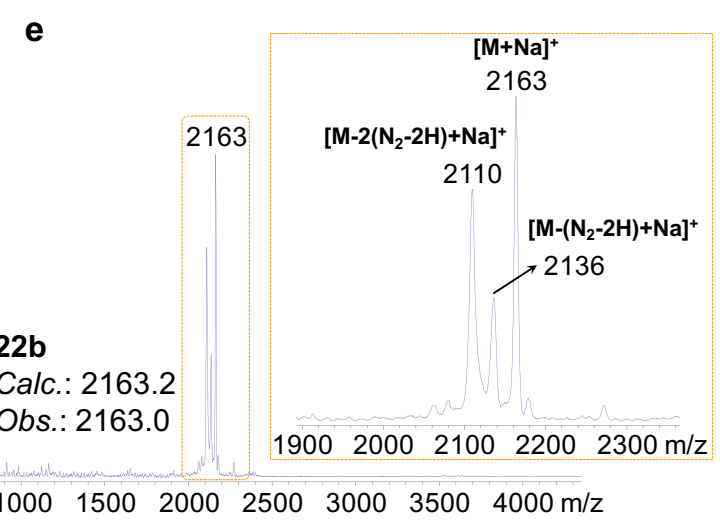

Fig. 5 | Cleavage of sequence-defined polyethers. a, The arms of the polyether-stars (21) were cleaved from the hub to provide sequencedefined polyethers (22). b, HPLC trace of LOLLOL-polyether (22a). c, HPLC trace of LONDON-polyether (22b). d, MALDI-ToF-MS of 22a. e, MALDI-ToF-MS of 22b. 
In order to complete the synthesis of sequence-defined polyethers, the polymers contained in the final polyetherstars (21a and 21b) must be cleaved from the hub. Initially palladium-catalyzed hydrogenolysis was attempted ${ }^{50,51}$, but almost no product was obtained, presumably due to poisoning of the catalyst by sulfur in the Pmb-thioether ${ }^{52}$. Instead, treatment with $\mathrm{BCl}_{3}$ in dichloromethane at $-78^{\circ} \mathrm{C}$ released the polyethers from the hub ${ }^{50,53}$, and simultaneously deprotected the benzyl ethers to give two sequence-defined polyethers (22a and 22b) on $>100 \mathrm{mg}$ scale (Fig. 5a). The molecular structures of 22a and 22b were confirmed by NMR spectrometry (see SI). In Figs. 5b-c, the resulting polyethers (22a and 22b) showed single HPLC peaks, demonstrating high purity and uniform nature, as further confirmed by the presence of single peaks and sharp molecular weight distribution in their GPC traces (Supplementary Fig. 16-2). MALDI-ToF-MS further confirmed the identity of 22a and 22b (Figs 5d-e). The observed $\mathrm{m} / \mathrm{z}$ values (2490.0 for 22a and 2163.0 for 22b) of the expected molecular ions $[\mathrm{M}+\mathrm{Na}]^{+}$were in good agreement with the theoretical molar masses of 2489.2 and $2163.2 \mathrm{Da}$, respectively. Two fragmentation peaks for [M$\left.n\left(\mathrm{~N}_{2}-2 \mathrm{H}\right)+\mathrm{Na}\right]^{+}(n=1-2)$ were also observed owing to the dissociation of two azide groups. The molecular sequences of 22a and 22b were further verified by tandem mass spectrometry (MS/MS) (Supplementary Figs 12-1 and 12-2). The thermal properties of 22a and 22b were evaluated by DSC and TGA. Both 22a and 22b are viscous oils at room temperature and each have a sharp $T_{g}$ but no detectable melting point $\left(T_{m}\right)$ (Supplementary Fig. 17-1), verifying their amorphous nature. This is consistent with Johnson's observations ${ }^{10}$. The difference in $\mathrm{T}_{\mathrm{g}}$ between $22 \mathrm{a}\left(-55.1^{\circ} \mathrm{C}\right)$ and 22b $\left(-50.3{ }^{\circ} \mathrm{C}\right)$ showed the effect of monomer sequence on their thermodynamic behaviors. The TGA traces in Supplementary Fig. 17-2 revealed their favorable thermal stability.

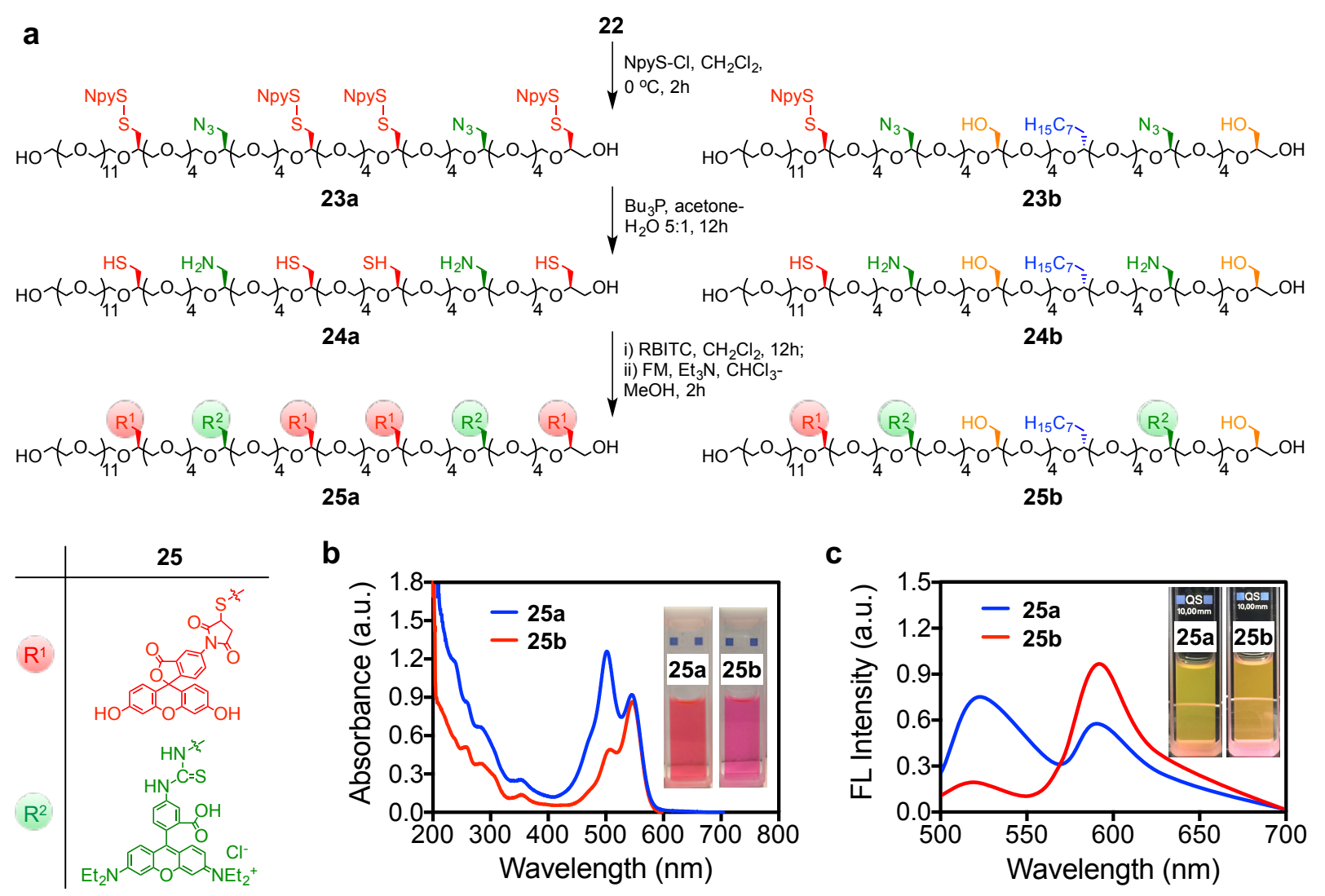

Fig. 6 | Deprotection and multi-functionalisation of sequence-defined polyethers. a, The side-arms of 22 were fully deprotected to give sequence-defined polyethers (24), followed by site-selective one-pot multifunctionalisation to yield two different sequences, dye-labelled LOLLOL (25a) and LONDON (25b). b, Steady-state absorption spectra (arbitrary units, a.u.) of 25a and 25b (Conc. $=5 \mu \mathrm{M})$; inset, photographs of methanolic solutions of 25a (Left) and 25b (Right). c, Steady-state fluorescence (FL) spectra of 25a and 25b, $\lambda_{\mathrm{Ex}}=490 \mathrm{~nm}$ $($ Conc. $=5 \mu \mathrm{M})$; inset, fluorescent photographs of methanolic solutions of 25a (Left) and 25b (Right). 
To demonstrate the versatility this new class of polyethers, we conducted site-selective post-synthetic modification. Reaction of each of 22a and 22b with 3-nitro-2-pyridylsulfenyl chloride (NpyS-Cl) converted the Pmbthioether groups to labile disulfides (23a and $\mathbf{2 3 b}$ ) (Fig. 6a) ${ }^{54}$. The partially deprotected intermediates were subsequently treated with tributyl phosphine in aqueous acetone to afford the fully-deprotected polyethers (24a and 24b) (Fig. 6a). The deprotection of both Pmb-thioether and azide side-arms was accomplished in $c a$. 80\% overall yield, over two steps from 22. Notably, these two fully-deprotected polyethers (24a and 24b) are highly soluble in water, with dissolution providing transparent aqueous solutions, demonstrating the potential for wide-ranging biomedical applications (Supplementary Fig. 18-1). Further, the reactive fluorophores rhodamine B isothiocyanate (RBITC) and fluorescein-5-maleimide (FM) were successively conjugated to the side-arm functionalities, in one pot to avoid losses during intermediate purification, to produce sequence-defined multifunctional polyethers (25a and $\mathbf{2 5 b}$, see SI). The resultant dye-labelled sequence-defined polyethers (25a and $\mathbf{2 5 b}$ ) exhibited significant rises in $\mathrm{T}_{\mathrm{g}}$ compared with 22a and $\mathbf{2 2} \mathbf{b}$, and by comparison the 25a had a much higher $T_{g}\left(+14.2{ }^{\circ} \mathrm{C}\right)$ than that of $25 \mathbf{b}\left(-32.4{ }^{\circ} \mathrm{C}\right)$, which might be attributed to a higher content of bulky functional dyes in 25a (Supplementary Fig. 17-1). The variation in optical behaviour with composition of the pendant side-arms of the two resulting polyethers was evaluated by UV-Vis and fluorescence spectroscopy. Both 25a and 25b showed UV-Vis absorption bands at $502 \mathrm{~nm}$ and 546 $\mathrm{nm}$, corresponding to the absorptions of fluorescein and rhodamine B, respectively (Fig. 6b). The FM-thioether-rich 25a gave a red-orange solution in methanol, with absorbance at $502 \mathrm{~nm}>546 \mathrm{~nm}$. In contrast, these intensities were reversed in the pink methanolic solution of RB-carbamate-rich $\mathbf{2 5 b}$. When the fluorescein residues of $\mathbf{2 5 a}$ were excited at $490 \mathrm{~nm}$, the polyether exhibited a strong green emission band from its four fluorescein fluorophores centered on $518 \mathrm{~nm}$, plus a relatively weak yellow emission band centered on $585 \mathrm{~nm}$ (Fig. 6c). While RB-carbamate-rich 25b showed only weak emission at $518 \mathrm{~nm}$ and strong emission at $585 \mathrm{~nm}$. The latter band arose because of Förster resonance energy transfer (FRET) from fluorescein to rhodamine B, suggesting that the majority of the excitation energy from the fluorescein donors was transferred to rhodamine B acceptors, resulting in overall yellow fluorescence. The difference in FRET between 25a and 25b is mainly associated with the different molar ratios of fluorescein donors to rhodamine B acceptors, i.e. 4:2 and 1:2, respectively. The combined data indicate that both side-chain structure and monomer sequence have a great impact on the thermal and optical properties of these sequence-defined polyethers.

\section{Conclusion}

We have demonstrated that a unique liquid phase synthetic approach combined with molecular sieving and real time monitoring can be successfully applied to production of high purity sequence-defined synthetic polyethers with readily tailored side-chain functionalities. This strategy takes advantage of real time monitoring to ensure reaction completion, and size-exclusion molecular sieving for purification in the liquid phase, to improve the sequence accuracy, purity and yield of target polymers. Site-selective post-synthetic modification then provides a versatile pathway to control precisely side-chain structures and functions. The demonstrated sequence accuracy, purity, yield, and potential scalability, as well as tunable functionalities, represent new benchmarks in the field of sequence-defined polymer synthesis. We expect that the molecular sieving-assisted liquid-phase synthesis platform will enable the creation of new kinds of sequence-defined polymers and their use in real-life applications.

\section{Methods}

General protocol for chain-extension. After evaporation from anhydrous $\mathrm{MeCN}$, hydroxyl-terminated polyether-star and selected BB tosylate (6-12 equiv.) were re-dissolved in DMF. To this solution was added $\mathrm{NaH}$ (6 equiv.). The resulting mixture was stirred for $12-24 \mathrm{~h}$ at $60{ }^{\circ} \mathrm{C}$ until complete, confirmed by HPLC. Saturated $\mathrm{NH}_{4} \mathrm{Cl}$ (aq.) was added, and solvent evaporated in vacuo. The residue was re-dissolved in $\mathrm{CH}_{2} \mathrm{Cl}_{2}$ and 
partitioned with half-saturated brine, back-extracting the aqueous layer. The combined $\mathrm{CH}_{2} \mathrm{Cl}_{2}$ layers were dried $\left(\mathrm{NaSO}_{4}\right)$, and the solvent stripped off. The crude material was diafiltered in $\mathrm{MeCN}-\mathrm{MeOH}$ (1:1), using a two-stage PBI-CC membrane apparatus. After 5 h, the retentate was washed from the apparatus and evaporated to provide the newly extended Thp-terminated polyether-star.

General protocol for Thp deprotection. To Thp-terminated polyether-star in $\mathrm{CH}_{2} \mathrm{Cl}_{2}-\mathrm{MeOH}(1: 1)$ was added $\mathrm{MsOH}(0.05 \mathrm{M})$. After 30 min, the reaction was quenched with saturated $\mathrm{NaHCO}_{3}$ (aq.) and the solvent evaporated. The residue was dissolved in $\mathrm{CHCl}_{3}$, partitioned with halfsaturated $\mathrm{NaHCO}_{3}$ (aq.), and back-extracted with further $\mathrm{CHCl}_{3}$. The combined organic phase was dried with $\mathrm{Na}_{2} \mathrm{SO}_{4}$ and concentrated at reduced pressure to afford the hydroxyl-terminated polyether-star.

NMR. ${ }^{1} \mathrm{H}$ NMR, ${ }^{13} \mathrm{C}$ NMR, 2D-COSY, 2D-HMBC and 2D-HSQC spectra were acquired using a Brüker AVANCE III-400 spectrometer, with working frequencies of $400\left({ }^{1} \mathrm{H}\right)$ and $101\left({ }^{13} \mathrm{C}\right) \mathrm{MHz}$, in $\mathrm{CDCl}_{3}$ or $\mathrm{CD}_{3} \mathrm{OD}$ at $293 \mathrm{~K}$.

ES-MS \& MALDI-ToF-MS. ES-MS were recorded on a Waters LCT Premier (ES-ToF)/Acquity i-Class mass spectrometer. MALDI-ToF-MS were conducted on a Brüker ultrafleXtreme MALDI-ToF/ToF mass spectrometer (with an Nd:YAG laser, $\lambda=355 \mathrm{~nm}$ ) using 1,8-dihydroxy$9(10 \mathrm{H})$-anthracenone (Dithranol) as the matrix. A typical sample preparation protocol was as follows: The samples and the matrix (Dithranol) were dissolved in $\mathrm{MeCN}$ or THF at $10 \mathrm{mg} / \mathrm{mL}$ and $20 \mathrm{mg} / \mathrm{mL}$, respectively, then mixed together in typically a 1:49 ratio to ensure an excess of matrix. $0.5 \mu \mathrm{L}$ of $10 \mathrm{mg} / \mathrm{mL} \mathrm{NaOAc}$ in $\mathrm{MeOH}$ was also added to promote $\mathrm{Na}$ adducts. Once mixed, $0.5 \mu \mathrm{L}$ of the mixture was spotted onto the MALDI plate. The samples were air-dried, then run in reflection or linear mode.

MS/MS. The MS/MS experiments of the resulting sequence-defined polyethers were conducted using nano-electrospray ionization on a Thermo Scientific LTQ Orbitrap XL with an Advion TriVersa NanoMate as the nano-electrospray ionization source. The samples were dissolved in $\mathrm{MeOH}$ containing $10 \mathrm{mM} \mathrm{NH}_{4} \mathrm{OAc}$. Optimisation of signal intensity was performed for the doubly charged ammonium adduct. The MS/MS spectra were acquired using collision induced dissociation (CID) fragmentation.

HPLC. HPLC analysis was performed on a 1100 Series Agilent HPLC system equipped with a UV diode array detector using a reverse-phase C4-300 column $(250 \mathrm{~mm} \times 4.6 \mathrm{~mm}$, ACE Hichrom). The mobile phases for HPLC were deionized water buffered with $5 \mathrm{mM}$ ammonium acetate (solvent A) and methanol (solvent B). The samples were injected using an Agilent 1100 Series (G1367A) autosampler with a capacity for 69 samples, and the injected volume was $30 \mu \mathrm{L}$. The samples were eluted with $70 \%$ solvent B for 2 min, followed by a linear gradient to $95 \%$ solvent B over $10 \mathrm{~min}$, and 95\% solvent B for $18 \mathrm{~min}$ before equilibrating the column back to $70 \%$ solvent B over $5 \mathrm{~min}$. The HPLC pump flow rate was set at $1 \mathrm{~mL} \mathrm{~min}^{-1}$ and the column temperature was kept at $30^{\circ} \mathrm{C}$. The detector was an Agilent 1100 Series (G1314A) variable wavelength detector and the eluent was monitored at 260, 280, 290 and $300 \mathrm{~nm}$.

Liquid-liquid porometry. Liquid-liquid porometry was used to avoid distorting the pore structure, or damaging the membranes. The membrane sample was initially soaked in a first wetting liquid (isobutanol-saturated water) to fill the pores, then a second wetting liquid (water-saturated isobutanol) displaced the first wetting liquid from the pores of the membrane under applied pressure, at an incremental rate of 5 bar min $^{-1}$ from 100 bar to 400 bar. The flow rate of the second wetting liquid through the emptied pores of the membrane was measured as a function of differential pressure, and also measured without the first wetting liquid in the membrane pores. The pore structure characteristics can then be computed from the measured differential pressures and flow rates of the second wetting liquid through the dry sample and sample wetted by the first wetting liquid.

UV-Vis absorption spectrometry. The UV-Vis absorption spectra were recorded on a Thermo Electron-EV300 UV-Vis spectrophotometer. The slit-width was set as $1 \mathrm{~nm}$, and scan speed was set as $480 \mathrm{~nm} \mathrm{~min}{ }^{-1}$. The wavelength range was from 200 to $700 \mathrm{~nm}$.

Steady-state fluorescence spectrometry. Fluorescence emission spectra were collected on a FluoroMax-4 fluorescence detector (HORIBA Jobin Yvon). The excitation wavelength of the samples was set at $490 \mathrm{~nm}$. The slit-width was set as $2 \mathrm{~nm}$, and the scan speed was set as $480 \mathrm{~nm}$ $\min ^{-1}$. The fluorescence range was from 500 to $700 \mathrm{~nm}$.

SEC. SEC analyses were performed on a Shimadzu Prominence GPC System with three mixed-bed GPC columns in series (two TSKgel 
SuperHZM-M and one TSKgel SuperHZ1000), and DMF with $0.1 \mathrm{wt} . \% \mathrm{LiBr}$ mobile phase run at $60^{\circ} \mathrm{C}$. The differential refractive index (dRI) of each compound was monitored using a Shimadzu's RID-10A detector.

DSC. DSC was performed on a TA Instruments Discovery DSC, where each sample was run with a Tzero aluminum pan sealed with a hermetic lid. Determination of the glass transition temperature was taken from the 2 nd heating cycle of a run where the sample was cycled at a rate of $10{ }^{\circ} \mathrm{C}$ per minute from -80 to $80^{\circ} \mathrm{C}$.

TGA. Thermal characterization of samples was performed on a TA Instruments Discovery TGA. The samples were run in platinum sample pans at a heating rate of $10{ }^{\circ} \mathrm{C}$ per minute from 50 to $600^{\circ} \mathrm{C}$.

\section{Data availability}

All data that support the findings of this study are included within the Article and its Supplementary Information, and are also available from the authors upon request.

\section{References}

1. Seeman, N. C. DNA in a material world. Nature 421, 427-431 (2003).

2. Van Hest, J. C. M. \& Tirrell, D. A. Protein-based materials, toward a new level of structural control. Chem. Commun. 1897-1904 (2001).

3. Zamfir, M. \& Lutz, J.-F. Ultra-precise insertion of functional monomers in chain-growth polymerizations. Nat. Commun. 3, 1138 (2012).

4. Srichan, S., Mutlu, H., Badi, N. \& Lutz, J.-F. Precision PEGylated polymers obtained by sequence-controlled copolymerization and postpolymerization modification. Angew. Chem. Int. Ed. 53, 9231-9235 (2014).

5. Nakatani, K., Ogura, Y., Koda, Y., Terashima, T. \& Sawamoto, M. Sequence-regulated copolymers via tandem catalysis of living radical polymerization and in situ transesterification. J. Am. Chem. Soc. 134, 4373-4383 (2012).

6. Pfeifer, S. \& Lutz, J.-F. A facile procedure for controlling monomer sequence distribution in radical chain polymerizations. J. Am. Chem. Soc. 129, 9542-9543 (2007).

7. Engelis, N. G. et al. Sequence-controlled methacrylic multiblock copolymers via sulfur-free RAFT emulsion polymerization. Nat. Chem. 9, 171-178 (2017).

8. McHale, R., Patterson, J. P., Zetterlund, P. B. \& O’Reilly, R. K. Biomimetic radical polymerization via cooperative assembly of segregating templates. Nat. Chem. 4, 491-497 (2012).

9. Lutz, J.-F. Sequence-controlled polymerizations: the next Holy Grail in polymer science? Polym. Chem. 1, 55-62 (2010).

10. Barnes, J. C. et al. Iterative exponential growth of stereo-and sequence-controlled polymers. Nat. Chem. 7, 810-815 (2015).

11. Lutz, J.-F., Ouchi, M., Liu, D. R. \& Sawamoto, M. Sequence-controlled polymers. Science 341, 1238149 (2013).

12. Solleder, S. C., Schneider, R. V., Wetzel, K. S., Boukis, A. C. \& Meier, M. A. R. Recent progress in the design of monodisperse, sequencedefined macromolecules. Macromol. Rapid Commun. 38, 1600711 (2017).

13. Merrifield, R. B. Solid phase synthesis. Science 232, 341-347 (1986).

14. Plante, O. J., Palmacci, E. R. \& Seeberger, P. H. Automated solid-phase synthesis of oligosaccharides. Science 291, 1523-1527 (2001).

15. Svec, F. \& Fréchet, J. M. J. New designs of macroporous polymers and supports: from separation to biocatalysis. Science 273, 205-211 (1996).

16. Paynter, O. I., Simmonds, D. J. \& Whiting, M. C. The synthesis of long-chain unbranched aliphatic-compounds by molecular doubling. J. Chem. Soc. Chem. Commun. 1165-1166 (1982).

17. Bidd, I. \& Whiting, M. C. The synthesis of pure n-paraffins with chain-lengths between one and four hundred. J. Chem. Soc. Chem. Commun. 543-544 (1985).

18. Pfeifer, S., Zarafshani, Z., Badi, N. \& Lutz, J.-F. Liquid-phase synthesis of block copolymers containing sequence-ordered segments. $J$. Am. Chem. Soc. 131, 9195-9197 (2009).

19. Roy, R. K. et al. Design and synthesis of digitally encoded polymers that can be decoded and erased. Nat. Commun. 6, 7237 (2015). 
20. Szweda, R., Chendo, C., Charles, L., Baxter, P. N. W. \& Lutz, J.-F. Synthesis of oligoarylacetylenes with defined conjugated sequences using tailor-made soluble polymer supports. Chem. Commun. 53, 8312-8315 (2017).

21. Takizawa, K., Tang, C. \& Hawker, C. J. Molecularly defined caprolactone oligomers and polymers: synthesis and characterization. $J$. Am. Chem. Soc. 130, 1718-1726 (2008).

22. Takizawa, K., Nulwala, H., Hu, J., Yoshinaga, K. \& Hawker, C. J. Molecularly defined (L)-lactic acid oligomers and polymers: synthesis and characterization. J. Polym. Sci. Part A: Polym. Chem. 46, 5977-5990 (2008).

23. Solleder, S. C. \& Meier, M. A. R. Sequence control in polymer chemistry through the Passerini three-component reaction. Angew. Chem. Int. Ed. 53, 711-714 (2014).

24. Solleder, S. C., Zengel, D., Wetzel, K. S. \& Meier, M. A. R. A scalable and high-yield strategy for the synthesis of sequence-defined macromolecules. Angew. Chem. Int. Ed. 55, 1204-1207 (2016).

25. Porel, M. \& Alabi, C. A. Sequence-defined polymers via orthogonal allyl acrylamide building blocks. J. Am. Chem. Soc. 136, 1316213165 (2014).

26. Porel, M., Thornlow, D. N., Phan, N. N. \& Alabi, C. A. Sequence-defined bioactive macrocycles via an acid-catalysed cascade reaction. Nat. Chem. 8, 590-596 (2016).

27. Oh, D., Ouchi, M., Nakanishi, T., Ono, H. \& Sawamoto, M. Iterative radical addition with a special monomer carrying bulky and convertible pendant: a new concept toward controlling the sequence for vinyl polymers. ACS Macro Lett. 5, 745-749 (2016).

28. Hibi, Y., Ouchi, M. \& Sawamoto, M. A strategy for sequence control in vinyl polymers via iterative controlled radical cyclization. Nat. Commun. 7, 11064 (2016).

29. Lewandowski, B. et al. Sequence-specific peptide synthesis by an artificial small-molecule machine. Science 339, 189-193 (2013).

30. Bootwicha, T., Feilner, J. M., Myers, E. L. \& Aggarwal, V. K. Iterative assembly line synthesis of polypropionates with full stereocontrol. Nat. Chem. 9, 896-902 (2017).

31. Niu, J, Hili, R. \& Liu, D. R. Enzyme-free translation of DNA into sequence-defined synthetic polymers structurally unrelated to nucleic acids. Nat. Chem. 5, 282-292 (2013).

32. Amir, F., Jia, Z. \& Monteiro, M. J. Sequence control of macromers via iterative sequential and exponential growth. J. Am. Chem. Soc. 138, 16600-16603 (2016).

33. Bayer, E. \& Mutter, M. Liquid phase synthesis of peptides. Nature 237, 512-513 (1972).

34. Harris, J. M. (ed.) Poly(ethylene glycol) Chemistry: Biotechnical and Biomedical Applications (Springer, 1992).

35. Knop, K., Hoogenboom, R., Fischer, D. \& Schubert, U. S. Poly(ethylene glycol) in drug delivery: pros and cons as well as potential alternatives. Angew. Chem. Int. Ed. 49, 6288-6308 (2010).

36. French, A. C., Thompson, A. L. \& Davis, B. G. High-purity discrete PEG-oligomer crystals allow structural insight. Angew. Chem Int. Ed. 48, 1248-1252 (2009).

37. Maranski, K., Andreev, Y. G. \& Bruce, P. G. Synthesis of poly(ethylene oxide) approaching monodispersity. Angew. Chem. Int. Ed. 53, 6411-6413 (2014).

38. Székely, G., Schaepertoens, M., Gaffney, P. R. J. \& Livingston, A. G. Iterative synthesis of monodisperse PEG homostars and linear heterobifunctional PEG. Polym. Chem. 5, 694-697 (2014).

39. Zhang, H. et al. Highly efficient synthesis of monodisperse poly(ethylene glycols) and derivatives through macrocyclization of oligo(ethylene glycols). Angew. Chem. Int. Ed. 54, 3763-3767 (2015).

40. Khanal, A. \& Fang, S. Solid phase stepwise synthesis of polyethylene glycols. Chem. Eur. J. 23, 15133-15142 (2017).

41. Karan, S., Jiang, Z. \& Livingston, A. G. Sub-10 nm polyamide nanofilms with ultrafast solvent transport for molecular separation. Science 348, 1347-1351 (2015).

42. Jimenez-Solomon, M. F., Song, Q., Jelfs, K. E., Munoz-Ibanez, M. \& Livingston, A. G. Polymer nanofilms with enhanced microporosity by interfacial polymerization. Nat. Mater. 15, 760-767 (2016).

43. Marchetti, P., Jimenez Solomon, M. F., Szekely, G. \& Livingston, A. G. Molecular separation with organic solvent nanofiltration: a 
critical review. Chem. Rev. 114, 10735-10806 (2014).

44. Koros, W. J. \& Zhang, C. Materials for next-generation molecularly selective synthetic membranes. Nat. Mater. 16, $289-297$ (2017).

45. König, N. F., Al Ouahabi, A., Poyer, S., Charles, L. \& Lutz, J.-F. A simple post-polymerization modification method for controlling sidechain information in digital polymers. Angew. Chem. Int. Ed. 56, 7297-7301 (2017).

46. Schaepertoens, M., Gaffney, P. R. J., Székely, G. \& Livingston, A. G. Process for preparing polymers. Patent No. WO2016020696 (A1) (2016).

47. da Silva Burgal, J., Peeva, L., Marchetti, P. \& Livingston, A. G. Controlling molecular weight cut-off of PEEK nanofiltration membranes using a drying method. J. Membrane Sci. 493, 524-538 (2015).

48. Gaffney, P. R. J., Livingston, A. G., Chen, R. \& Dong, R. Defined monomer sequence polymers. Patent No. WO2017042583 (A1) (2017).

49. Kim, J. F., Freitas da Silva, A. M., Valtcheva, I. B. \& Livingston, A. G. When the membrane is not enough: a simplified membrane cascade using organic solvent nanofiltration (OSN). Sep. Purif. Technol. 116, 277-286 (2013).

50. Weissman, S. A. \& Zewge, D. Recent advances in ether dealkylation. Tetrahedron 61, 7833-7863 (2005).

51. Nishimura, S. (ed.) Handbook of Heterogeneous Catalytic Hydrogenation for Organic Synthesis 572-663 (Wiley-Interscience, 2001).

52. Dunleavy, J. Sulfur as a catalyst poison. Platinum Metals Rev. 50, 110 (2006).

53. Okaya, S., Okuyama, K., Okano, K. \& Tokuyama, H. Trichloroboron-promoted deprotection of phenolic benzyl ether using pentamethylbenzene as a non-Lewis basic cation scavenger. Org. Synth. 93, 63-74 (2016).

54. Matsueda, R., Higashida, S., Ridge, R. J. \& Matsueda, G. R. Activation of conventional S-protecting groups of cysteine by conversion into the 3-nitro-2-pyridinesulfenyl (Npys) group. Chem. Lett. 921-924 (1982).

\section{Acknowledgements}

This work was financially supported by the Engineering and Physical Sciences Research Council (EPSRC, EP/M003949/1) and GlaxoSmithKline (GSK, UK). The authors acknowledge the EPSRC UK National Mass Spectrometry Facility at Swansea University for the MALDI-ToF-MS measurement. The authors thank Robert T. Woodward for the GPC analysis, and Chunyang Yu for the molecular modelling.

\section{Author contributions}

R.D., P.R.J.G., R.C. and A.G.L. conceived the project. R.D., P.R.J.G. and A.G.L. designed the experiments. R.D. carried out the synthesis and characterisation of BnO-BB, $\mathrm{N}_{3}$-BB, PmbS-BB and polymers, and analysed the data. R.L. synthesised Octyl-BB. R.D. conducted the DSC, TGA, UV-Vis and fluorescence spectrometry measurements. R.D. and R.L. carried out organic solvent nanofiltration. R.L. performed liquid-liquid porometry and membrane screening. M.S. provided the hub molecule. P.M. prepared the PBI membranes. C.M.W. performed the MS/MS measurement. R.D. and P.R.J.G. wrote the manuscript. A.G.L. guided the project. All of the authors discussed the results and edited the manuscript.

\section{Competing interests}

The Imperial Innovations has filed a UK Patent application (No. 1516067.4) related to defined monomer sequence polymers leading to PCT/GB2016/052801. A.G.L., P.R.J.G., R.D., R.C., P.M. and R.L. are listed as inventors. All other authors declare no competing interests.

\section{Additional information}

Supplementary information is available for this paper at https://doi.org/10.1038/xxxxxxxx.

Reprints and permissions information is available at www.nature.com/reprints.

Correspondence and requests for materials should be addressed to A.G.L.

Publisher's note: Springer Nature remains neutral with regard to jurisdictional claims in published maps and institutional affiliations. 